\title{
Article
}

\section{Comparison of Two EpCAM-Based Methods for CTC Detection and Molecular Characterization in Advanced Colorectal Cancer}

\section{Santiago Cabezas-Camarero ${ }^{1}$, Virginia De La Orden ${ }^{2}$, Silvia Veganzones-De-Castro 2 , Beatriz Medievo-Valeros ${ }^{2}$, Manuel Enrique Fuentes-Ferrer ${ }^{3}$, Antonio Sánchez-Ruiz ${ }^{4}$, Mariano Provencio ${ }^{4}$, Enrique Aranda ${ }^{5}$, Javier Sastre ${ }^{1}$ and Eduardo Díaz-Rubio ${ }^{1, *}$}

1. Medical Oncology Department, Instituto de Investigación Sanitaria San Carlos (IdISSC), Hospital Clínico Universitario San Carlos, Centre afilliated to Red de Centros CIBER, 28040 Madrid, Spain; santicc81@gmail.com (S.C.-C.); jsastrev@salud.madrid.org (J.S.)

2. CTC Unit, Hospital Clínico Universitario San Carlos, 28040 Madrid, Spain; virginia.delaorden@gmail.com (V.D.L.O.-G.); silvia_veg@yahoo.es (S.V.-D.-C.); bea.medierovaleros@gmail.com (B.M.-V.)

3. UGC de Medicina Preventiva, Hospital Clínico San Carlos, Instituto de Investigación Sanitaria del Hospital Clínico San Carlos (IdISSC), 28040 Madrid, Spain; mfuentesferrer@gmail.com

4. Medical Oncology Department, Hospital Universitario Puerta de HierroMajadahonda, IDIPHIM, Universidad Autónoma de Madrid, 28049 Madrid, Spain; acsr71@hotmail.com (A.S.-R.); mprovencio.hpth@salud.madrid.org (M.P.)

5. Medical Oncology Department, IMIBIC, Hospital Universitario Reina Sofía, Córdoba, University of Córdoba, RETICC, 14002 Córdoba, Spain; earandaa@seom.org

* Correspondence: eduardo.diazrubio@salud.madrid.org; Tel.: +34-913-303-546

Abstract: Circulating tumor cells (CTC) have shown to be prognostic in advanced colorectal cancer (advCRC), but their value for predicting response to treatment or as a source of molecular data is debated. We compared CellSearch ${ }^{\circledR}$ (Janssen Diagnostics, LLC) and IsoFlux ${ }^{\mathrm{TM}}$ (Fluxion Biosciences Inc, South San Francisco, CA) systems for the enumeration of CTC in patients with newly diagnosed $\operatorname{advCRC}$ (group $1 ; n=34$ ). Using castPCR ${ }^{\mathrm{TM}}$ we studied KRAS status in CTC isolated with IsoFlux ${ }^{\mathrm{TM}}$ and compared it with that of the primary tumor in patients from group 1 and in KRAS wild-type (KRASWT) patients with progressive disease (group 2; $n=22$ ). Median number of CTC detected with CellSearch ${ }^{\circledR}$ (groups 1 and 2) was 1 (range: $0-78$ ) and with IsoFlux ${ }^{\mathrm{TM}}$ (group 1 ) was 
8 (range: 0-419), showing a modest correlation ( $r=0.345, P=0.036)$, which improved if lung metastases $(r=0.805, P=0.016)$ or if lung and liver metastases were present $(r=0.812, P=0.05)$. A Bland-Altman plot showed that the higher the number of CTC detected the larger the difference between both methods in favor of IsoFlux ${ }^{\mathrm{TM}}$. After a median follow-up since CTC collection of 16 months (range: 1 -30) CellSearch ${ }^{\circledR} \geq 3$ CTC (HR 2.77, 95\% Cl 0.77-9.95) and IsoFlux ${ }^{\mathrm{TM}} \geq 11 \mathrm{CTC}$ (HR 4.14, 95\% Cl 1.05-16.19) were established as the best cutoff points for predicting survival. Using castPCR ${ }^{\mathrm{TM}}$ we found KRAS mutations in CTC in 4 out of 8 patients from group 1 and in 2 out of 3 patients from group 2. None of these mutations were found in the primary tumor using standard methods, possibly reflecting intratumor heterogeneity or treatment selection pressure. We conclude that IsoFlux ${ }^{\mathrm{TM}}$ is more efficient than CellSearch ${ }^{\circledR}$ in the isolation of CTC in patients with advCRC, achieving, in a majority of cases, the established minimum of CTC for castPCR ${ }^{\mathrm{TM}}$-based genetic analyses.

Keywords: circulating tumor cells; CTC, liquid biopsy; KRAS; colorectal cancer; EpCAM; CellSearch; IsoFlux; castPCR; intratumor heterogeneity 


\section{Introduction}

Traditional biopsies provide a static picture of a certain tumor area, which may not be the most relevant in terms of metastatic potential and resistance to treatments. Furthermore, they may be troublesome to obtain, and carry important morbidities and costs [1], which dot not allow for their serial performance. Liquid biopsies are intended to circumvent these limitations through highly refined technologies that easily detect different biomarkers shed by tumors into the systemic circulation, such as circulating tumor cells (CTC), circulating tumor DNA (ctDNA), or exosomes among others $[\mathbf{2}, \mathbf{3}]$. Elevated CTC counts with the CellSearch ${ }^{\circledR}$ technology indicate a poor prognosis in breast, prostate, lung and colorectal cancers [4-13]. However, most CTC technologies do not recover enough number of CTC to perform molecular studies, therefore precluding CTC from becoming also a predictive tool. Indeed, the CellSearch ${ }^{\circledR}$ system, an epithelial cell adhesion molecule (EPCAM)-based immunomagnetic enrichment method, and sole FDA-approved CTC technology to date, does not allow for the recovery of CTC to perform molecular analyses $[2,14]$. IsoFlux ${ }^{\mathrm{TM}}$, a novel microfluidic device that uses single or multiple capture antibodies -usually targeting EpCAM- for CTC isolation, was designed to achieve maximum recovery efficiency and cell transferability to provide sufficient CTC for molecular studies [14]. The IsoFlux ${ }^{\mathrm{TM}}$ system has demonstrated a high isolation effiency in locally advanced bladder cancer, and in advanced prostate, renal, CRC and non-small cell lung cancers, as well as in patients with hepatocarcinoma awaiting for a liver transplant [14-19]. Furthermore, in bladder, prostate and renal cancers IsoFlux ${ }^{\mathrm{TM}}$ was able to recover enough cells for performing molecular analyses through droplet digital PCR (ddPCR) and Next Generation Sequencing (NGS) [15-17]. Liquid biopsies are expected to offer real-time 
data on the molecular dynamics of cancer, and this may prove specially useful in cancers with known molecular alterations with predictive and/or prognostic value [20]. Colorectal cancer (CRC), which stands as the third most common cancer and fourth cause of mortality worldwide, is also one of the few malignancies with a predictive molecular marker (RAS status) that aids in the selection of the most appropriate treatment option [21]. any-RAS (KRAS or NRAS) mutant tumors constitute aproximately $50 \%$ of advanced CRC ( $\operatorname{advCRC)}$ cases, $80 \%$ of which are due to mutations in KRAS [22]. all-RAS wild-type patients show an improved survival with chemotherapy plus anti-epidermal growth factor receptor (anti-EGFR) antibodies and thus benefit from more treatment options than RAS mutated patients [23]. While RAS mutational analysis and other molecular studies have been performed on ctDNA from advCRC patients, showing promise as predictive tools, few CTC technologies are claimed to efficiently recover enough cells to allow for subsequent molecular studies [24]. Therefore, we conducted a prospective-retrospective study to test the enumeration efficiency of IsoFlux ${ }^{\mathrm{TM}}$ and compared it to the current standard-CellSearch ${ }^{\circledR}-$. Finally, we tested three different Polymerase Chain Reaction (PCR)-based methods to perform KRAS mutational analysis on CTC previously recovered through IsoFlux ${ }^{\mathrm{TM}}$. 


\section{Results}

\section{a. Patient characteristics at diagnosis and at CTC collection}

A total of 56 patients were included in the analysis. Thirty-four were assigned to group 1 -chemo-naïve patients- and 22 to group 2 - KRAS wild-type (KRAS ${ }^{\mathrm{WT}}$ ) patients progressing to chemotherapy with or without anti-EGFR or antivascular endothelial growth factor (VEGF) agents. Twenty-nine were male and 27 female. Median age at diagnosis was 67 years (range: 41-84). A majority of patients had advanced disease at diagnosis (66\%), with liver, lung and lymphnodes being the most common sites of metastasis $(86.5 \%, 24 \%$ and $24 \%$, respectively). Surgical resection of the primary had been performed in $79 \%$ of the patients. Sixteen out of 18 patients diagnosed in stages II or III had received adjuvant chemotherapy. Surgery for distant metastases had been performed in $36.5 \%$ of the patients.

At CTC collection, liver, lung and lymph-node metastases were present in $70 \%$, $28 \%$ and $21 \%$, respectively, and $13 \%$ had peritoneal carcinomatosis. At CTC collection $68 \%$ and $46.8 \%$ had carcinoembryonic antigen (CEA) and CA 19.9 values above their reference cut-off points $(>5 \mathrm{ng} / \mathrm{ml}$ and $>35 \mathrm{U} / \mathrm{ml}$ ), respectively.

In group $1,54.5 \%$ were KRAS $^{\mathrm{WT}}$ and $45.5 \%$ were KRAS mutated (KRAS ${ }^{\mathrm{MUT}}$ ). Among the 22 patients from group 2 -all KRAS ${ }^{W T}$ - at the time of progression prior to CTC collection, 9 patients (41\%) were receiving chemotherapy with anti-VEGF agents, 5 patients (23\%) were receiving anti-EGFR agents -either combined with chemotherapy or as monotherapy-, 4 patients (18\%) chemotherapy alone, and 4 patients (18\%) were on treatment holidays. 
Most clinico-pathological characteristics were well balanced between the two groups and are summarized in Table 1.

Table 1. Summary of patient characteristics.

\begin{tabular}{|c|c|c|c|c|c|c|c|c|c|}
\hline & & \multicolumn{4}{|c|}{ Group 1} & \multicolumn{4}{|c|}{ Group 2} \\
\hline \multicolumn{2}{|l|}{$\mathbf{N}$} & \multicolumn{4}{|c|}{34} & \multicolumn{4}{|c|}{22} \\
\hline \multicolumn{2}{|c|}{ Gender (male:female) } & \multicolumn{4}{|c|}{$14: 20$} & \multicolumn{4}{|c|}{$15: 7$} \\
\hline \multicolumn{2}{|c|}{ Age at CTC determination (median) } & \multicolumn{4}{|c|}{$71(44-84)$} & \multicolumn{4}{|c|}{$64(45-81)$} \\
\hline \multicolumn{2}{|c|}{ AJCC Stage at diagnosis } & $\begin{array}{c}\mathrm{I}: \\
2.9 \%\end{array}$ & II: $8.8 \%$ & $\begin{array}{c}\text { III: } \\
20.6 \% \\
\end{array}$ & $\begin{array}{l}\text { IV: } \\
67.6 \% \\
\end{array}$ & $\begin{array}{l}\mathrm{I}: \\
0 \%\end{array}$ & $\begin{array}{l}\text { II: } \\
9.1 \% \\
\end{array}$ & $\begin{array}{c}\text { III: } \\
27.3 \% \\
\end{array}$ & $\begin{array}{c}\text { IV: } \\
63.6 \%\end{array}$ \\
\hline \multirow{3}{*}{$\begin{array}{l}\text { Primary tumor } \\
\text { location }\end{array}$} & Left colon & \multicolumn{4}{|c|}{$38.2 \%(23.5 \%$ sigma $)$} & \multicolumn{4}{|c|}{$37 \%$ (23\% sigma) } \\
\hline & Right colon & \multicolumn{4}{|c|}{$32.4 \%$} & \multicolumn{4}{|c|}{$29 \%$} \\
\hline & Rectum & \multicolumn{4}{|c|}{$29.4 \%$} & \multicolumn{4}{|c|}{$34 \%$} \\
\hline \multicolumn{2}{|c|}{ Resection of primary tumor } & \multicolumn{4}{|c|}{$80 \%$} & \multicolumn{4}{|c|}{$77 \%$} \\
\hline \multirow{2}{*}{\multicolumn{2}{|c|}{ Histologic type }} & \multicolumn{4}{|c|}{ Mucinous: $12.1 \%$} & \multicolumn{4}{|c|}{ Mucinous: $12.5 \%$} \\
\hline & & \multicolumn{4}{|c|}{ NOS: $87.9 \%$} & & & S: $87.5 \%$ & \\
\hline & Grade 1 & & & & & & & $35.7 \%$ & \\
\hline Histologic grade & Grade 2 & & & & & & & $57.1 \%$ & \\
\hline & Grade 3 & & & & & & & $7.1 \%$ & \\
\hline Adjuvant & & & $6 / 11$ & $55 \%)$ & & & & $8(62.5 \%)$ & \\
\hline Surgery for distant & metastases & & & & & & & $38.1 \%$ & \\
\hline $\begin{array}{l}\text { No. of CT lines for ad } \\
\text { (median }\end{array}$ & anced disease & & & & & & & $3(1-7)$ & \\
\hline & 1st line & & & & & & & $100 \%$ & \\
\hline $\begin{array}{l}\text { Fluoropyrimidine- } \\
\text { hased CT }\end{array}$ & 2nd line & & & & & & & $80 \%$ & \\
\hline & 3rd line & & & & & & & $92 \%$ & \\
\hline Anti-EGFR agents & Any time & & & & & & & $100 \%$ & \\
\hline & Prior to CTC & & & & & & & $77 \%$ & \\
\hline Anti-VEGF agents & Any time & & & & & & & $82 \%$ & \\
\hline & Prior to CTC & & & & & & & $73 \%$ & \\
\hline & Wild-type & & $18 / 33$ & $54.5 \%)$ & & & & $22(100 \%)$ & \\
\hline $\begin{array}{l}\text { KRAS } \\
\text { (nrimarv tumor) }\end{array}$ & Mutated & & $15 / 33$ & $45.5 \%)$ & & & & - & \\
\hline & Unknown & & & & & & & - & \\
\hline No of CTC (median) & CellSearch ${ }^{\circledR}$ & & & 78) & & & & $(0-17)$ & \\
\hline No. or CIC (mealan) & IsoFlux ${ }^{\mathrm{TM}}$ & & 810 & 419) & & & & - & \\
\hline & Liver & & & & & & & $68.4 \%$ & \\
\hline & Lung & & & & & & & $36.8 \%$ & \\
\hline metastases at CTC & $\mathrm{LN}$ & & & & & & & $31.6 \%$ & \\
\hline collection & Peritoneum & & & & & & & $10.5 \%$ & \\
\hline & Local & & & & & & & $15.8 \%$ & \\
\hline & Other & & & & & & & $5.3 \%$ & \\
\hline CEA $\geq 5$ at CTC C & llection & & & & & & & $64.7 \%$ & \\
\hline CA $19.9 \geq 35$ at CT & collection & & & & & & & $47.1 \%$ & \\
\hline
\end{tabular}

Group 1: patients chemo-naïve for advanced disease at the time of CTC collection; Group 2: KRAS wild-type patients with progressive disease at the time of CTC collection. AJCC: American Joint Committee on Cancer, CT: chemotherapy, CTC: circulating tumor cells, EGFR: epidermal growth factor receptor, LN: lymph nodes, N/A: not applicable, NOS: not otherwise specified, VEGF: vascular endothelial growth factor. 


\section{b. CTC enumeration, association with clinical characteristics and correlation}

with survival

\section{i. $\quad$ CTC enumeration with CellSearch ${ }^{\circledR}$ and IsoFlux ${ }^{\mathrm{TM}}$.}

CTC were counted both with CellSearch ${ }^{\circledR}$ and IsoFlux ${ }^{\mathrm{TM}}$ in 34 from group 1. Median number of CTC detected with CellSearch ${ }^{\circledR}$ was 1 (range: 0-78) and with IsoFlux ${ }^{\mathrm{TM}}$ was 8 (range: 0-419) (Figure 1) (Table

Table 3. Association and correlation of different clinical characteristics with CTC counts for CellSearch ${ }^{\circledR}$ and IsoFlux ${ }^{\mathrm{TM}}$.

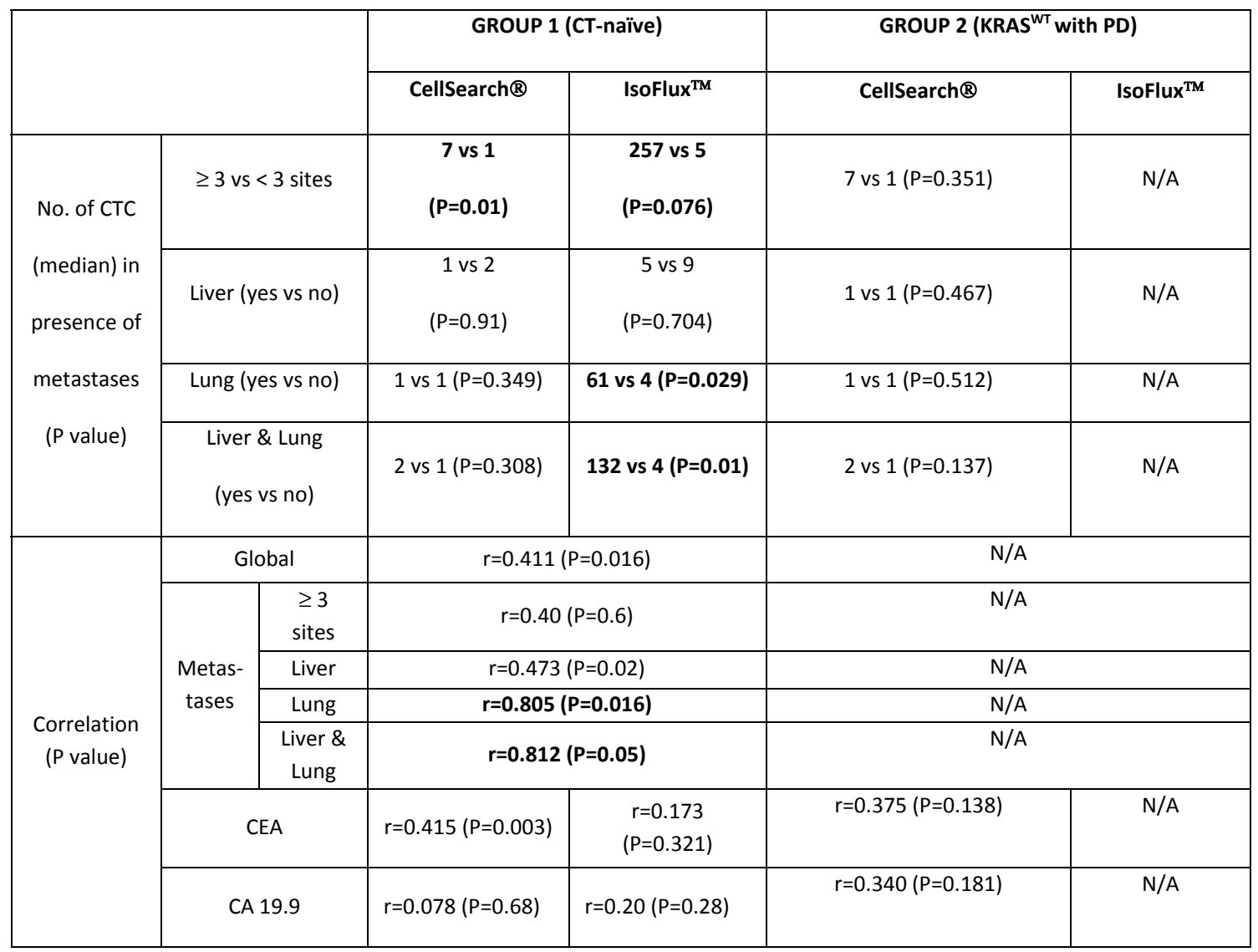

Association of different clinical characteristics with CTC counts with CellSearch ${ }^{\circledR}$ and IsoFlux ${ }^{\mathrm{TM}}$. Group 1: patients chemo-naïve for advanced disease at CTC collection; Group 2: KRAS wild-type patients with progressive disease at CTC collection. CTC: circulating tumor cells. For more information, please refer to the text.

2). 
$1 a$

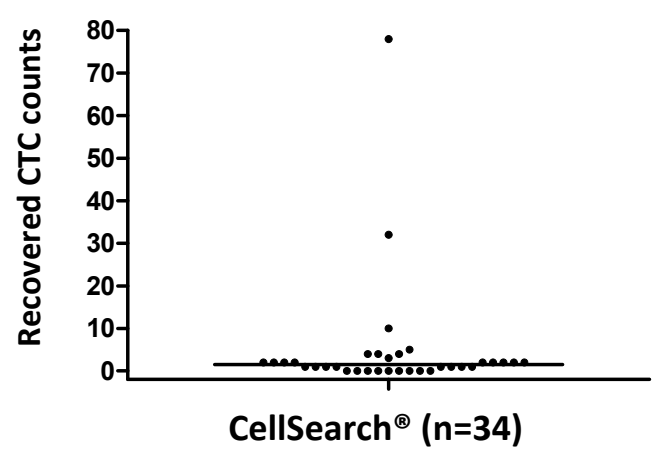

$1 b$

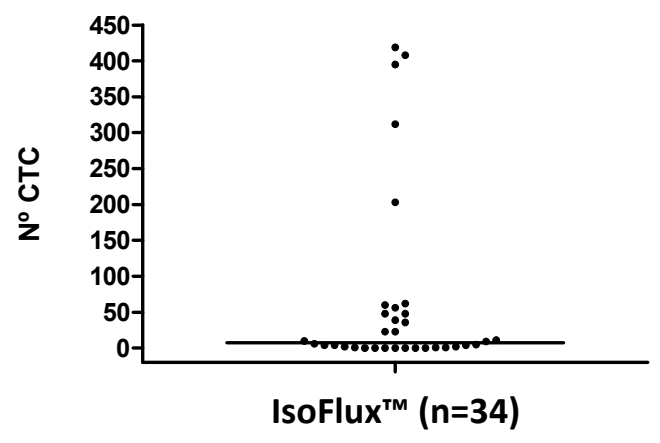

1c

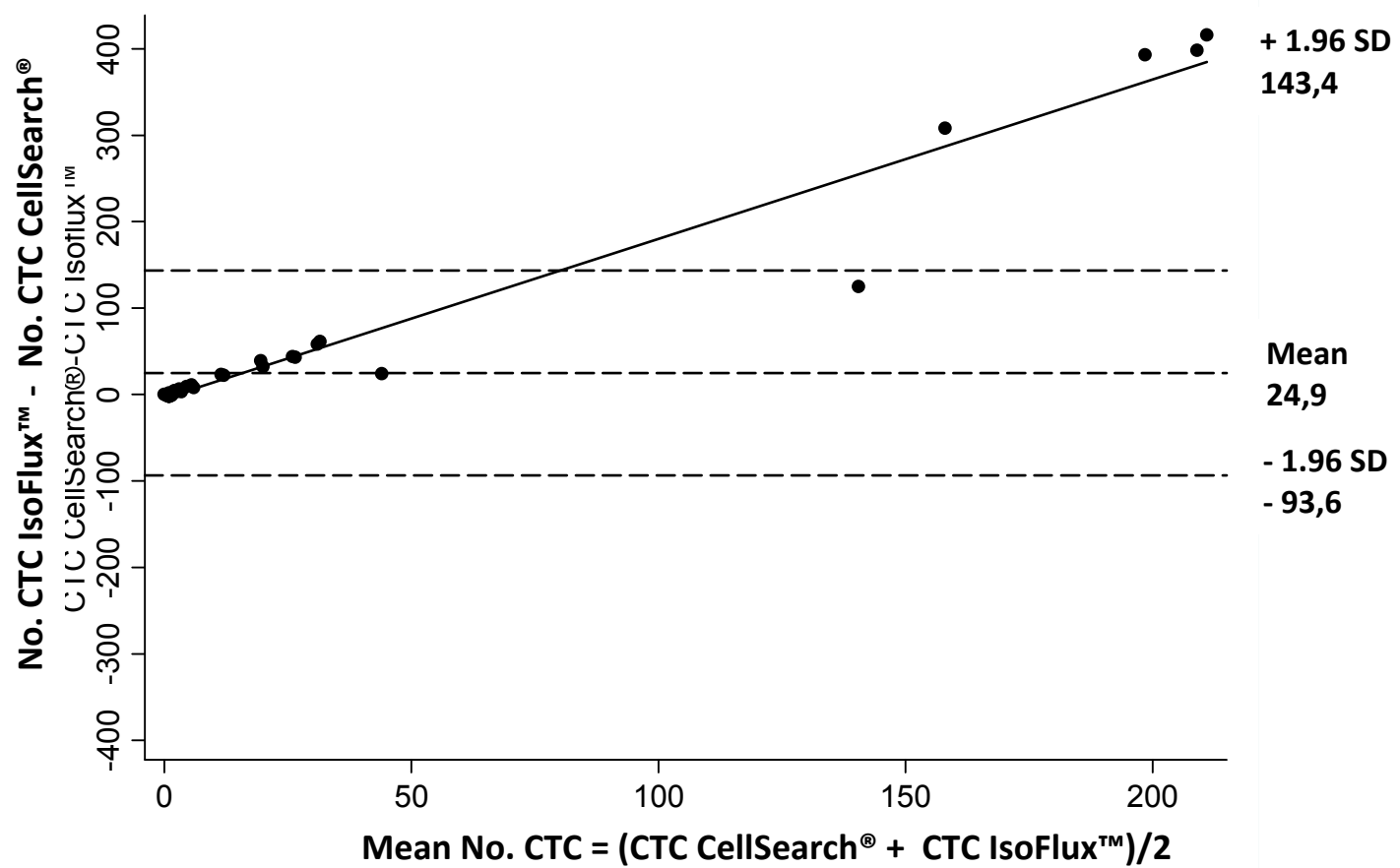

Figure 1. Number of CTC detected with CellSearch ${ }^{\circledR}$ (1a) and IsoFlux ${ }^{\mathrm{TM}}$ (1) $\left.\mathbf{1}\right)$ in patients from group 1. Solid line represents the median. Please note that the scale is different for each detection system. Bland-Altman plot showing the agreement between CellSearch ${ }^{\circledR}$ and IsoFlux ${ }^{\mathrm{TM}}$ in the detection of CTC in group 1 (1c). At higher CTC number detected with IsoFlux ${ }^{\mathrm{TM}}$ the larger the difference compared to CellSearch $^{\circledR}$. 


\section{ii. Correlation and agreement between CellSearch ${ }^{\circledR}$ and IsoFlux ${ }^{\mathrm{TM}}$}

CellSearch $\AA$ and IsoFlux ${ }^{\mathrm{TM}}$ showed a modest correlation $(r=0.411$, $P=0.016)$ for the enumeration of CTC. The same ocurred when there were $\geq 3$ metastatic locations $(r=0.4, P=0.6)$ and in the presence of hepatic metastases $(r=0.473, P=0.020)$. Correlation improved with pulmonary metastases $(r=0.805, P=0.016)$, and when both pulmonary and hepatic metastases coexisted $(r=0.812, P=0.05)$. There was a modest correlation between CellSearch $\AA$ and CEA $(r=0.415, P=0.003)$ and between both tumor markers $(r=0.542, P=0.0001)$. No significant correlations between IsoFlux ${ }^{\mathrm{TM}}$ and CEA $(r=0.17, \mathrm{P}=0.32)$ or CA 19.9 $(r=0.16, P=0.39)$ were found (Table 2).

In order to test the agreement between the two enumeration systems, we constructed a Bland-Altman plot, which shows that the higher the number of CTC detected with IsoFlux ${ }^{\mathrm{TM}}$ the larger the difference for CTC enumeration compared to CellSearch ${ }^{\circledR}$ (Figure 1).

\section{iii. Survival and definition of cut-off points for CTC}

After a median follow-up since CTC collection of 16 months (range: 126 ) in group 1 and 13.4 months (range: $2-30$ ) in group 2, median survival was NR for group 1, and achieved 15.7 months (range: 2-30) in group 2 (Figure 2). 

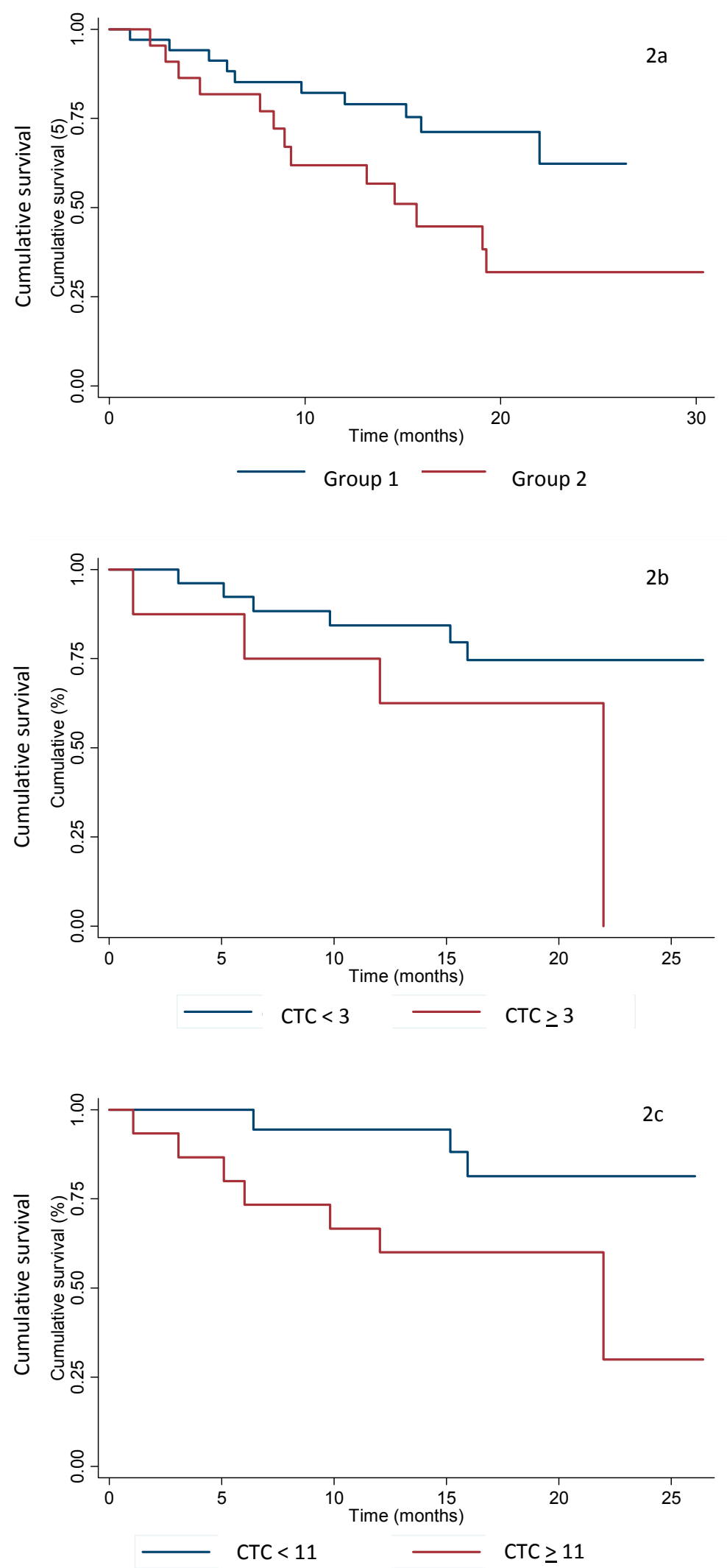

Figure 2. Overall survival curves in both groups from time of CTC collection (2a), and depending on selected cut-off points for each enumeration system. Cut-off points $\geq 3$ CTC for CellSearch ${ }^{\circledR}$ (2b) and $\geq 11$ CTC for IsoFlux ${ }^{\mathrm{TM}}$ (2c) predicted significantly worse outcomes. 
Using the area under ROC curves and Youden's test, CTC $\geq 3$ with CellSearch ${ }^{\circledR}(\mathrm{HR} 2.77,95 \% \mathrm{Cl} 0.77-9.95)$ and $\mathrm{CTC} \geq 11$ with IsoFlux ${ }^{\mathrm{TM}}$ (HR 4.14, 95\% Cl 1.05-16.19) were identified as the best cut-off points for predicting survival in group 1. In order to test the discrimination of the model, Harrell's C-statistic was calculated. The highest C-statistic values in group 1 ocurred for CellSearch $® \geq 3$ CTC (c=0.59) and IsoFlux ${ }^{\mathrm{TM}}$ $\geq 11$ CTC (c=0.68), meaning that in group 1 IsoFlux ${ }^{\mathrm{TM}} \geq 11$ CTC was a slightly better predictor of survival than CellSearch $® \geq 3$ CTC but without reaching statistical significance $(P=0.379)$ (Table 3).

Table 3. Sensitivity, specificity and Harrell's C statistic for different CTC cut-off points for CellSearch ${ }^{\circledR}$ and IsoFlux ${ }^{\mathrm{TM}}$ in patients from group 1.

\begin{tabular}{|c|c|c|c|c|c|c|}
\hline \multicolumn{3}{|c|}{ CTC cut-off } & Sensitivity & Specificity & HR $(95 \% \mathrm{Cl})$ & C statistic \\
\hline \multirow{13}{*}{ Group 1} & \multirow{4}{*}{ CellSearch $®$} & $\mathrm{CTC} \geq 1$ & $80 \%$ & $29.17 \%$ & $1.42(0.29-6.73)$ & 0.50 \\
\hline & & $\mathrm{CTC} \geq 2$ & $60 \%$ & $54.17 \%$ & $1.65(0.46-5.88)$ & 0.54 \\
\hline & & CTC $\geq 3$ & $40 \%$ & $83.33 \%$ & $2.77(0.77-9.95)$ & 0.59 \\
\hline & & $\mathrm{CTC} \geq 4$ & $30 \%$ & $83.33 \%$ & $2.54(0.62-10.35)$ & 0.59 \\
\hline & \multirow{9}{*}{ IsoFlux ${ }^{\mathrm{TM}}$} & $\mathrm{CTC} \geq 4$ & $70 \%$ & $37.50 \%$ & $1.67(0.43-6.51)$ & 0.57 \\
\hline & & $\mathrm{CTC} \geq 5$ & $70 \%$ & $50 \%$ & $2.37(0.60-9.22)$ & 0.62 \\
\hline & & $\mathrm{CTC} \geq 6$ & $70 \%$ & $54.2 \%$ & $2.49(0.64-9.65)$ & 0.63 \\
\hline & & $\mathrm{CTC} \geq 7$ & $70 \%$ & $58.3 \%$ & $3.01(0.77-11.70)$ & 0.65 \\
\hline & & $\mathrm{CTC} \geq 8$ & $70 \%$ & $58.3 \%$ & $3.01(0.77-11.70)$ & 0.65 \\
\hline & & CTC $\geq 9$ & $70 \%$ & $58.3 \%$ & $3.01(0.77-11.70)$ & 0.65 \\
\hline & & CTC $\geq 10$ & $70 \%$ & $62.5 \%$ & $3.70(0.94-14.54)$ & 0.67 \\
\hline & & CTC $\geq 11$ & $70 \%$ & $66.7 \%$ & 4.14 (1.05-16.19) & 0.68 \\
\hline & & CTC $\geq 12$ & $60 \%$ & $66.7 \%$ & $2.70(0.76-9.67)$ & 0.62 \\
\hline
\end{tabular}

The best cut-off points for each enumeration system are shown in bold. Group 1: patients chemo-naïve for advanced disease at CTC collection; CTC: circulating tumor cells. For more information, please refer to the text. 
iv. Association of CTC number and CTC cut-off points with clinical characteristics

Median number of CTC with CellSearch ${ }^{\circledR}$ was significantly higher in the presence of $\geq 3$ metastatic locations ( $\geq 3$ vs $<3: 7$ vs 1 CTC, P=0.01) and showed a trend towards significance for $\operatorname{IsoFlux}^{\mathrm{TM}}$ ( $\geq 3$ vs $<3: 257$ vs 5 CTC, $\mathrm{P}=0.076)$. The number of $\mathrm{CTC}$ with IsoFlux ${ }^{\mathrm{TM}}$ was also significantly higher when there were lung metastases (Yes vs No: 61 vs 4 CTC, $P=0.029)$. There was no difference in the number of CTC with either collection system for the presence or absence of hepatic metastases and for CEA or CA 19.9 levels (Table 2 and Figure 3). In patients from group

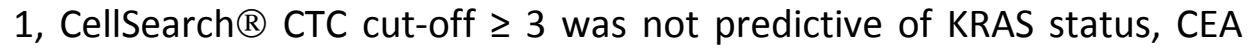
and CA 19.9 levels, and neither of hepatic or pulmonary metastases. For IsoFlux ${ }^{\mathrm{TM}}$ CTC cut-off $\geq 11$, more patients had pulmonary metastases (CTC $\geq 11$ vs $<11: 40 \%$ vs $10.5 \%, P=0.10$ ) and CA 19.9 levels above normal (CTC $\geq 11$ vs $<11: 69.2 \%$ vs $29.4 \%, P=0.03$ ) (Table 4 ). As an exploratory analysis in patients from group 2 , we studied the influence of prior biological agents on CTC count with CellSearch ${ }^{\circledR}$. The median number of CTC did not change depending on prior treatment or not with either anti-EGFR (yes vs no: 1 vs 1 CTC) or anti-VEGF agents (yes vs no: 1 vs 1 CTC). 

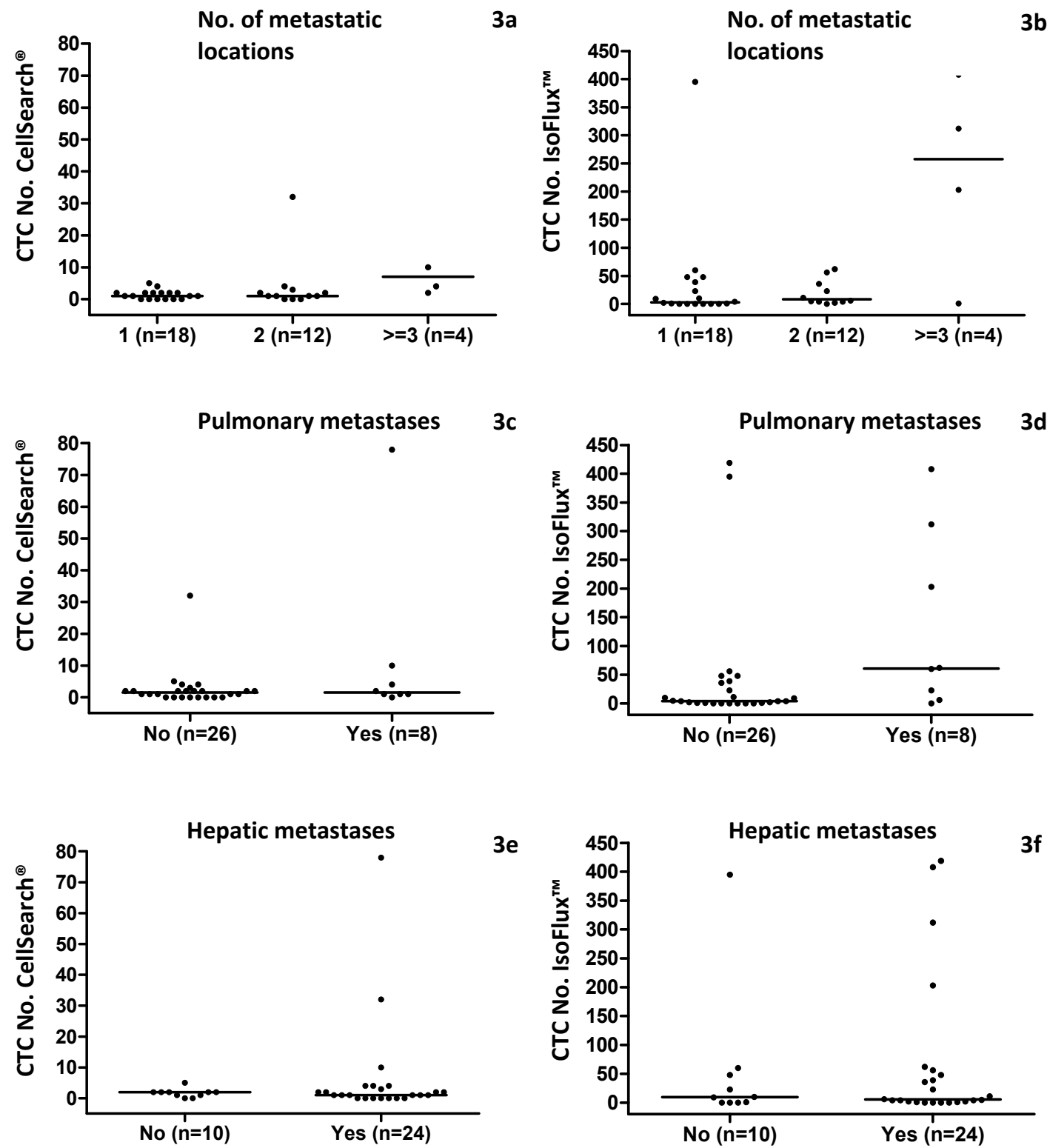

Figure 3. Number of CTC depending on the number of metastatic locations, and presence of pulmonary or hepatic metastases in patients from group 1 (Patients chemo-naïve for advanced disease at CTC collection). With $\geq 3$ metastatic sites vs $<3$, the number of CTC detected with CellSearch ${ }^{\circledR}$ was significantly higher (3a). IsoFlux ${ }^{\mathrm{TM}}$ also detected more CTC but without reaching statistical significance (3b). There were no differences in CTC count with CellSearch ${ }^{\circledR}(3 \mathrm{c})$ for lung metatases, but median number of CTC with IsoFlux ${ }^{\mathrm{TM}}$ was significantly higher if they were present (3d). No differences were found in CTC count with either enumeration system regarding hepatic metastases (3e, $\mathbf{3 f}$ ). Solid lines represent the median. 
Table 4. Association of different clinical characteristics with selected CTC cutoffs for CellSearch $\AA$ and IsoFlux ${ }^{\mathrm{TM}}$ in patients form group 1.

\begin{tabular}{|c|c|c|c|c|c|c|}
\hline \multicolumn{3}{|c|}{ Group 1} & \multicolumn{2}{|c|}{$\begin{array}{l}\text { CellSearch }{ }^{\circledR} \\
\geq 3 \text { vs } 3 \text { CTC }\end{array}$} & \multicolumn{2}{|c|}{$\begin{array}{c}\text { IsoFlux }{ }^{\mathrm{TM}} \\
\geq 11 \text { vs }<11\end{array}$} \\
\hline \multirow{2}{*}{$\begin{array}{c}\text { KRAS } \\
\text { primary tumor }\end{array}$} & \multicolumn{2}{|c|}{ WT } & 50 vs & \multirow{2}{*}{$P=1.0$} & $\begin{array}{c}53.3 \% \\
\text { vs }\end{array}$ & \\
\hline & \multicolumn{2}{|c|}{ MUT } & $\begin{array}{l}50 \text { vs } \\
44 \%\end{array}$ & & $\begin{array}{c}46.7 \% \\
\text { vs } \\
44.4 \%\end{array}$ & \\
\hline \multirow{2}{*}{ Sex } & \multicolumn{2}{|c|}{ Male } & $\begin{array}{l}50 \text { vs } \\
38.5 \%\end{array}$ & \multirow{2}{*}{$P=0.7$} & $\begin{array}{c}40.0 \% \\
\text { vs }\end{array}$ & \\
\hline & \multicolumn{2}{|c|}{ Female } & $\begin{array}{l}50 \text { vs } \\
61.5 \%\end{array}$ & & $\begin{array}{l}\text { vs } \\
57.9 \%\end{array}$ & \\
\hline \multirow{7}{*}{ Metastases } & \multicolumn{2}{|c|}{$\geq 3$ sites } & $\begin{array}{l}37.5 \\
\text { vs }\end{array}$ & \multirow{3}{*}{$P=0.038$} & $\begin{array}{c}20.0 \% \\
\text { vs }\end{array}$ & \\
\hline & \multirow{2}{*}{\multicolumn{2}{|c|}{$<3$ sites }} & 62.5 & & $80.0 \%$ & $P=0.299$ \\
\hline & & & $\begin{array}{c}\text { vs } \\
96.2 \%\end{array}$ & & $\begin{array}{c}\text { vs } \\
94.7 \%\end{array}$ & \\
\hline & \multirow{2}{*}{ Liver } & Yes & $\begin{array}{c}87.5 \\
\text { vs } \\
65.4 \%\end{array}$ & \multirow{2}{*}{$\mathrm{P}=0.385$} & $\begin{array}{c}73.3 \% \\
\text { vs } \\
68.4 \%\end{array}$ & \multirow{2}{*}{$P=1.0$} \\
\hline & & No & $\begin{array}{c}12.5 \\
\text { vs } \\
34.6 \%\end{array}$ & & $\begin{array}{c}26.7 \% \\
\text { vs } \\
31.6 \%\end{array}$ & \\
\hline & \multirow{2}{*}{ Lung } & Yes & $\begin{array}{c}37.5 \\
\text { vs } \\
19.2 \%\end{array}$ & \multirow{2}{*}{$P=0.355$} & $\begin{array}{c}40.0 \% \\
\text { vs } \\
10.5 \%\end{array}$ & \multirow{2}{*}{$P=0.10$} \\
\hline & & No & $\begin{array}{c}62.5 \\
\text { vs } \\
80.8 \%\end{array}$ & & $\begin{array}{c}60.0 \% \\
\text { vs } \\
89.5 \%\end{array}$ & \\
\hline \multirow{2}{*}{ CEA } & \multicolumn{2}{|c|}{$\leq 5$} & $\begin{array}{c}14.3 \\
\text { vs } \\
34.6 \%\end{array}$ & \multirow{2}{*}{$P=0.397$} & $\begin{array}{c}35.7 \% \\
\text { vs } \\
26.3 \%\end{array}$ & \multirow{2}{*}{$\mathrm{P}=0.71$} \\
\hline & \multicolumn{2}{|c|}{$>5$} & $\begin{array}{c}85.7 \\
\text { vs } \\
65.4 \%\end{array}$ & & $\begin{array}{c}64.3 \% \\
\text { vs } \\
73.7 \%\end{array}$ & \\
\hline \multirow{2}{*}{ CA 19.9} & \multicolumn{2}{|c|}{$\leq 35$} & $\begin{array}{c}33.3 \\
\text { vs } \\
58.3 \%\end{array}$ & \multirow{2}{*}{$P=0.378$} & $\begin{array}{c}30.8 \% \\
\text { vs } \\
70.6 \%\end{array}$ & \multirow{2}{*}{$P=0.03$} \\
\hline & \multicolumn{2}{|c|}{$>35$} & $\begin{array}{c}66.7 \\
\text { vs } \\
41.7 \%\end{array}$ & & $\begin{array}{c}69.2 \% \\
\text { vs } \\
29.4 \%\end{array}$ & \\
\hline
\end{tabular}

More patients with CTC $\geq 3$ with CellSearch had $\geq 3$ metastatic locations compared to CTC $<3$. Likewise, significantly more patients with CTC $\geq 11$ with IsoFlux ${ }^{\mathrm{TM}}$ had CA 19.9 levels above normal. CEA: carcinoembryonic antigen. MUT: KRAS mutated. WT: KRAS wild-type. 


\section{c. KRAS status in the primary tumor and in CTC}

\section{i. KRAS status in primary tumor by real-time PCR (RT-PCR)}

KRAS status in the primary tumor could not be determined in one patient from group 1 due to insufficient sample. Among the remaining 33 cases, sixteen were KRAS ${ }^{\mathrm{WT}}$ and 15 were $\mathrm{KRAS}^{\mathrm{MUT}}$. As per protocol, all patients from group 2 had confirmed KRASWT tumors (Table 5).

ii. KRAS status in CTC using cobas ${ }^{\circledR}$ RT-PCR and CLART ${ }^{\circledR}$ CMA multiplex PCR (mPCR)

When using cobas ${ }^{\circledR}$ RT-PCR, no mutations were detected in CTC in 17 patients from group 1, even though 6 of them were KRAS ${ }^{\mathrm{MUT}}$ in the primary tumor. When using CLART $\AA$ CMA mPCR, all but 1 of the 9 patients analysed from group 1 where KRASWT in CTC, despite all being KRAS ${ }^{M U T}$ in the primary tumor.

All the 14 patients from group 2 analysed with cobas ${ }^{\circledR}$ turned out to be KRAS ${ }^{W T}$, even though all of them had received prior therapy with anti-EGFR agents. No CTC DNA from patients in group 2 was left for KRAS mutational analysis using MPCR (Table 5).

iii. KRAS status in CTC using Competitive Allele-Specific Taqman PCR (castPCR $^{\mathrm{TM}}$ )

KRAS status was analysed by castPCR ${ }^{\mathrm{TM}}$ in 8 patients from group 1 and in 3 patients from group 2. Compared to the primary tumor, KRAS changed its status in CTC in 3 and 2 patients, respectively. 
Seven of these patients were found to carry KRAS mutations in CTC, in 6 of them with a dCT $<21$, being, therefore, considered undoubtedly true mutations. The p.G13D and p.G12A mutations were the most common, while p.G12S and p.G12V were also detected. Moreover, in one patient, castPCR ${ }^{\mathrm{TM}}$ unveiled two different KRAS mutations, both with a dCt below 21 (p.G12A [10.7], p.G13D [dCt 12]). However, no coincidence was found between the type of mutation in the primary tumor and that in CTC in any of the patients. This discordance is probably explained by the low sensitivity of RT-PCR compared to castPCR ${ }^{\mathrm{TM}}$ and may reflect the intratumor heterogeneity. (Table 5).

Table 5. CTC count and KRAS status in the primary tumor and in CTC in patients from groups 1 and 2 .

\begin{tabular}{|c|c|c|c|c|c|c|}
\hline $\begin{array}{c}\text { Group } 1 \\
\text { (CT- } \\
\text { naïve) }\end{array}$ & \multicolumn{2}{|c|}{ CTC count } & $\begin{array}{c}\text { Primary } \\
\text { tumor }\end{array}$ & \multicolumn{3}{|c|}{ KRAS CTC } \\
\hline $\begin{array}{c}\text { Patient } \\
\text { No. }\end{array}$ & CellSearch $®$ & IsoFlux ${ }^{\mathrm{TM}}$ & $\begin{array}{c}\text { KRAS } \\
\text { RT-PCR }\end{array}$ & $\begin{array}{l}\text { cobas } \AA \\
\text { RT-PCR }\end{array}$ & $\begin{array}{c}\text { CLART }{ }^{8} \\
\text { mPCR }\end{array}$ & castPCR $^{\mathrm{TM}}$ \\
\hline 1 & 4 & 36 & $W^{a}$ & WT & - & - \\
\hline 2 & 2 & 0 & $W T^{a}$ & - & - & - \\
\hline \multirow[t]{2}{*}{3} & \multirow[t]{2}{*}{78} & \multirow[t]{2}{*}{203} & \multirow[t]{2}{*}{$W T^{a}$} & WT & - & $\begin{array}{c}\text { pG12A } \\
\text { [dCt 17.3] }\end{array}$ \\
\hline & & & & \multicolumn{3}{|c|}{ DNA concentration: $68.5 \mathrm{ng} / \mu \mathrm{l}$} \\
\hline 4 & 2 & 395 & $W T^{a}$ & WT & - & - \\
\hline \multirow[t]{2}{*}{5} & \multirow[t]{2}{*}{0} & \multirow[t]{2}{*}{6} & \multirow[t]{2}{*}{$\mathrm{pG} 12 \mathrm{~S}^{\mathrm{a}}$} & WT & WT & WT \\
\hline & & & & \multicolumn{3}{|c|}{ DNA concentration: $97.9 \mathrm{ng} / \mu \mathrm{l}$} \\
\hline 6 & 0 & 9 & $\mathrm{pG} 12 \mathrm{D}^{\mathrm{a}}$ & WT & WT & $\begin{array}{l}\text { pG13D } \\
\text { [dCt 18] }\end{array}$ \\
\hline
\end{tabular}




\begin{tabular}{|c|c|c|c|c|c|c|}
\hline 7 & 0 & 2 & (no sample) & - & - & - \\
\hline 8 & 1 & 1 & $\mathrm{pG} 12 \mathrm{D}^{\mathrm{a}}$ & - & WT & - \\
\hline 9 & 2 & 60 & MUT $^{b}$ & WT & - & $\begin{array}{l}\text { pG13D } \\
\text { [dCt 12] }\end{array}$ \\
\hline 10 & 0 & 0 & $\mathrm{WT}^{\mathrm{a}}$ & WT & - & - \\
\hline \multirow[t]{2}{*}{11} & \multirow[t]{2}{*}{1} & \multirow[t]{2}{*}{4} & \multirow[t]{2}{*}{$\mathrm{pG} 12 \mathrm{~V}^{\mathrm{a}}$} & WT & - & $\begin{array}{l}\mathrm{pG} 13 \mathrm{D} \\
\text { [dCt 33] }\end{array}$ \\
\hline & & & & \multicolumn{3}{|c|}{ DNA concentration: $63.8 \mathrm{ng} / \mu \mathrm{l}$} \\
\hline 12 & 5 & 48 & $\mathrm{pG} 12 \mathrm{C}^{\mathrm{a}}$ & WT & - & - \\
\hline 13 & 1 & 23 & $\mathrm{pG} 12 \mathrm{D}^{\mathrm{a}}$ & WT & - & - \\
\hline 14 & 2 & 0 & $W^{a}$ & - & - & - \\
\hline 15 & 4 & 48 & $\mathrm{WT}^{\mathrm{a}}$ & - & WT & - \\
\hline 16 & 0 & 23 & $W T^{a}$ & WT & - & - \\
\hline 17 & 2 & 0 & $\mathrm{WT}^{\mathrm{a}}$ & - & - & - \\
\hline 18 & 3 & 419 & MUT $^{b}$ & WT & MUT & - \\
\hline 19 & 1 & 2 & MUT $^{b}$ & WT & - & - \\
\hline 20 & 2 & 1 & $\mathrm{WT}^{\mathrm{a}}$ & - & - & - \\
\hline 21 & 0 & 4 & $\mathrm{MUT}^{\mathrm{b}}$ & WT & - & - \\
\hline 22 & 0 & 4 & MUT $^{\mathrm{b}}$ & - & WT & WT \\
\hline 23 & 0 & 11 & MUT $^{b}$ & WT & WT & - \\
\hline 24 & 1 & 0 & MUT $^{b}$ & - & WT & $\begin{array}{l}\mathrm{pG12V} \\
\text { [dCt 9] }\end{array}$ \\
\hline 25 & 2 & 0 & $W T^{b}$ & - & - & - \\
\hline 26 & 10 & 408 & $W T^{b}$ & WT & - & - \\
\hline 27 & 2 & 10 & $W T^{b}$ & WT & - & - \\
\hline 28 & 2 & 5 & $W T^{b}$ & WT & - & WT \\
\hline 29 & 1 & 0 & MUT $^{b}$ & - & - & - \\
\hline 30 & 4 & 312 & $\mathrm{MUT}^{\mathrm{b}}$ & - & - & - \\
\hline 31 & 32 & 56 & MUT $^{b}$ & - & - & - \\
\hline 32 & 1 & 62 & $W T^{a}$ & WT & - & - \\
\hline 33 & 1 & 1 & $\mathrm{WT}^{\mathrm{a}}$ & - & - & - \\
\hline 34 & 0 & 39 & $W^{a}$ & - & - & - \\
\hline $\begin{array}{l}\text { No. of } \\
\text { СTC }\end{array}$ & $1(0-78)$ & $8(0-419)$ & & & & \\
\hline
\end{tabular}




\begin{tabular}{|c|c|c|c|c|c|c|}
\hline $\begin{array}{l}\text { (median } \\
\text { \& range) }\end{array}$ & & & & & & \\
\hline $\begin{array}{c}\text { No. } \\
\text { mutated } \\
\text { (\%) }\end{array}$ & & & $16 / 33(49 \%)$ & $\begin{array}{l}0 / 19 \\
(0 \%)\end{array}$ & $\begin{array}{c}1 / 8 \\
(12.5 \%)\end{array}$ & $\begin{array}{c}5 / 8 \\
(62.5 \%)\end{array}$ \\
\hline $\begin{array}{l}\text { Group } 2 \\
\text { (KRASWT }^{\text {WT }} \\
\text { with PD) }\end{array}$ & CTC c & & $\begin{array}{l}\text { Primary } \\
\text { Tumor }\end{array}$ & & KRAS C & \\
\hline $\begin{array}{c}\text { Patient } \\
\text { No. }\end{array}$ & CellSearch $\circledast$ & IsoFlux ${ }^{\mathrm{TM}}$ & RT-PCR & $\begin{array}{l}\text { Cobas }{ }^{\circledR} \\
\text { RT-PCR }\end{array}$ & mPCR & castPCR $^{\mathrm{TM}}$ \\
\hline 1 & 0 & 65 & $\mathrm{WT}^{\mathrm{a}}$ & WT & - & - \\
\hline 2 & 0 & & $\mathrm{WT}^{\mathrm{a}}$ & & - & - \\
\hline 3 & 1 & & $W T^{b}$ & & - & - \\
\hline 4 & 0 & & $W T^{b}$ & WT & - & - \\
\hline 5 & 1 & & $W^{T^{a}}$ & WT & - & - \\
\hline 6 & 2 & & $\mathrm{WT}^{\mathrm{a}}$ & & - & - \\
\hline 7 & 4 & & $\mathrm{WT}^{\mathrm{a}}$ & & - & - \\
\hline 8 & 1 & 193 & $W T^{a}$ & WT & - & - \\
\hline 9 & 1 & 296 & $W T^{a}$ & WT & - & $\begin{array}{c}\text { pG12A [dCt } \\
19.4]\end{array}$ \\
\hline & & & & DNA & centratio & $1.5 \mathrm{ng} / \mu \mathrm{l}$ \\
\hline 10 & 1 & & $W T^{b}$ & WT & - & - \\
\hline 11 & 4 & & $W T^{b}$ & WT & - & - \\
\hline 12 & 12 & & $W T^{b}$ & WT & - & - \\
\hline 13 & 7 & & $W T^{b}$ & & - & - \\
\hline 14 & 1 & & $W T^{b}$ & & - & - \\
\hline 15 & 1 & & $W T^{b}$ & & - & - \\
\hline 16 & 0 & & $W T^{b}$ & & - & - \\
\hline 17 & 6 & & $W T^{b}$ & & - & - \\
\hline 18 & 0 & & $W T^{b}$ & WT & - & - \\
\hline 19 & 0 & & $W T^{b}$ & WT & - & - \\
\hline \multirow{3}{*}{20} & \multirow{3}{*}{2} & & \multirow{3}{*}{$\mathrm{WT}^{\mathrm{a}}$} & \multirow{2}{*}{ WT } & \multirow{2}{*}{ - } & $\begin{array}{c}\text { pG12A [dCt } \\
10.7 \text { ] }\end{array}$ \\
\hline & & & & & & $\begin{array}{c}\text { pG13D [dCt } \\
\text { 12] }\end{array}$ \\
\hline & & & & \multicolumn{3}{|c|}{ DNA concentration: $98 \mathrm{ng} / \mu \mathrm{l}$} \\
\hline
\end{tabular}




\begin{tabular}{|c|c|c|c|c|c|c|}
\hline 21 & 17 & & $W T^{b}$ & WT & - & WT \\
\hline 22 & 1 & - & $W T^{b}$ & & - & - \\
\hline $\begin{array}{l}\text { No. of } \\
\text { CTC } \\
\text { (median } \\
\text { \& range) }\end{array}$ & $1(0-17)$ & N/A & & & & \\
\hline $\begin{array}{l}\text { No. } \\
\text { mutated } \\
(\%)\end{array}$ & & & $0 / 22(0 \%)$ & $0 / 12(0 \%)$ & N/A & $2 / 3(67 \%)$ \\
\hline
\end{tabular}

Four patients from group 1 and 2 patients from group 2 were found to be KRAS mutated in CTC using castPCR ${ }^{\mathrm{TM}}$. Mutations with a $\mathrm{dCT}<21$ are considered undoubtedly true mutations. In another patient from group 1, a mutation in CTC was detected but with a $\mathrm{dCt}$ above $21(\mathrm{dCt}=33)$ making this result inconclusive. Group 1: patients chemo-naïve for advanced disease at CTC collection; Group 2: KRAS wild-type patients with PD (progressive disease) at CTC collection. castPCR $^{\mathrm{TM}}$ : Competitive Allele-Specific Taqman PCR, CTC: circulating tumor cells, mPCR: CLART ${ }^{\circledR}$

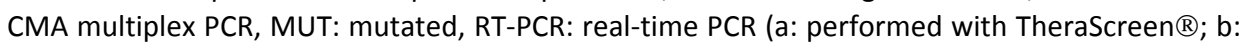
performed with cobas $\left.{ }^{\circledR}\right)$, UK: unknown, WT: wild-type. DNA concentration for KRAS mutational analyses on CTC is shown for cases were this information was available. For more details, please refer to the text. 


\section{Discussion}

We show that IsoFlux ${ }^{\mathrm{TM}}$ is a highly efficient technology for enumerating CTC. In a cohort of chemo-naïve patients with advCRC unselected for KRAS status (group 1), IsoFlux ${ }^{\mathrm{TM}}$ detected a median of $8 \mathrm{CTC}$, while CellSearch ${ }^{\circledR}$ isolated only 1 CTC. The median number of CTC isolated with CellSearch ${ }^{\circledR}$ was significantly higher with $\geq 3$ metastatic locations and showed a trend towards statistical significance with IsoFlux ${ }^{\mathrm{TM}}$. Moreover, in the presence of lung metastases, IsoFlux ${ }^{\mathrm{TM}}$ did detect significantly more CTC than CellSearch ${ }^{\circledR}$. Although we did not find a good correlation between the two systems, this was notably improved when there were lung metastases and when lung and hepatic metastases coexisted. Altoghether, these results suggest that both systems perform better with higher tumor burdens. We also tested the agreement between both CTC detection systems by means of a Bland-Altman plot, and show that at higher CTC counts the difference in detection eficiency with IsoFlux ${ }^{\mathrm{TM}}$ becomes wider compared to CellSearch ${ }^{\circledR}$ (Figure 1).

CTC $\geq 3$ is established as the best cutoff for predicting survival with CellSearch ${ }^{\circledR}$ in $\operatorname{advCRC}[\mathbf{7 , 8}]$, and we found a similar result in group 1 of our study (Figure $\mathbf{2}$ and Table 3). However, there is no established cutoff for IsoFlux ${ }^{\mathrm{TM}}$ in advCRC or in other malignancies [14-19]. In our sample (group 1) we found $\geq 11$ CTC as the best cutoff point for predicting survival with IsoFlux ${ }^{\mathrm{TM}}$, with patients below 11 CTC living significantly longer than those with $\geq 11$ CTC (Figure 2). Furthermore, Harrell's Cstatistic suggested that IsoFlux ${ }^{\mathrm{TM}} \geq 11$ CTC was at least as good as a predictor of survival as CellSearch ${ }^{\circledR} \geq 3$ CTC. However, prospective studies with a larger sample 
size should be conducted to confirm CTC $\geq 11$ as the best prognostic cutoff for IsoFlux ${ }^{\mathrm{TM}}$ in advCRC.

We performed an exploratory analysis in patients from group 2, and found that the number of CTC detected with CellSearch ${ }^{\circledR}$ did not change depending on prior treatment or not with anti-EGFR or anti-VEGF agents. The latter dissagrees with previous "in vitro" studies with a human CRC cell line, where treatment with bevacizumab increased the expression of the EpCAM $42 \mathrm{kDA}$ isoform decreasing that of the constitutive $40 \mathrm{kDA}$ isoform, and this correlated with a reduced CTC recovery rate with CellSearch®. In our study we only used IsoFlux ${ }^{\mathrm{TM}}$ for CTC enumeration in three patients from group 2, very few to derive conclusions on how treatment pressure may affect the isolation efficiency with this system. This is particularly important, since anti-EpCAM capture antibodies used by CellSearch ${ }^{\circledR}$ and IsoFlux ${ }^{\top M}$ may have structural differences that make the latter less susceptible to treatmentinduced changes in EpCAM isoforms expression [25]. In fact, again through spiking experiments, Bard et al [14], showed that IsoFlux ${ }^{\mathrm{TM}}$ detected significantly more CTC than CellSearch ${ }^{\circledR}$ when EpCAM expression was low. Although no data are yet available in CRC, IsoFlux ${ }^{\mathrm{TM}}$ can use other capture antibodies, such as anti-EGFR antibodies, alone or in combination with anti-EpCAM antibodies, to maximize isolation efficiency [26]. To our knowledge, it is currently unknown whether the capture efficiency of these antibodies used by IsoFlux ${ }^{\mathrm{TM}}$ can be altered by anti-EGFR or anti-VEGF agents.

The median number of CTC detected with CellSearch ${ }^{B}-1$ CTC- was well below the established limit of detection (LOD) for castPCR ${ }^{\mathrm{TM}}$, usually set at $\geq 4 \mathrm{CTC}$, for performing genetic analyses [14]. IsoFlux ${ }^{\mathrm{TM}}$, however, was able to isolate a median of 8 CTC and where CellSearch ${ }^{\circledR}$ detected $\geq 3$ CTC, IsoFlux ${ }^{\mathrm{TM}}$ always detected $\geq 30$ CTC. 
Indeed, IsoFlux ${ }^{\mathrm{TM}}$ detected $\geq 4$ CTC in $65 \%$ of our patients, while CellSearch ${ }^{\circledR}$ did so in $20.5 \%$ of the cases, a relative difference similar to what has been previously reported by the manufacturer in a small cohort of patients with prostate cancer [14]. Therefore, IsoFlux ${ }^{\mathrm{TM}}$ guarantees, in a majority of patients, a number of CTC well above the established LOD for castPCR ${ }^{\mathrm{TM}}$-based mutational studies. Likewise, other authors established a similar LOD ( $\geq 5$ CTC) when studying the androgen receptor variant 7 (ARV7) with droplet digital PCR (ddPCR) in CTC isolated with IsoFlux ${ }^{\mathrm{TM}}$ in prostate cancer [17].

In order to test if the higher efficiency in isolation achieved with IsoFlux ${ }^{\mathrm{TM}}$ actually allowed to perform genetic studies, we employed three different PCR-based methods for KRAS mutational analysis: cobas ${ }^{\circledR}$ RT-PCR, CLART ${ }^{\circledR}$ CMA multiplex PCR (mPCR) and castPCR ${ }^{\mathrm{TM}}$. These methods, are claimed to have $5 \%, \geq 1 \%$, and $0.5 \%$ sensitivity, respectively, for the detection of mutations in KRAS [27-30]. cobas ${ }^{\circledR}$ RT-PCR did not find any KRAS mutation in CTC among 31 patients tested from the two groups (17 from group 1 and 14 from group 2). CLART ${ }^{\circledR}$ CMA mPCR was tested in CTC samples from 9 KRAS ${ }^{M U T}$ patients from group 1 , finding only 1 patient KRAS ${ }^{M U T}$ in CTC. Due to the high discordance between the primary tumor KRAS status and that on CTC, these results do not seem reliable. Results using cobas ${ }^{\circledR}$ on CTC are not explained by low CTC numbers, since most had CTC counts well above the established LOD ( $\geq 4$ CTC) and many had, indeed, very high CTC counts. However, most of the few patients tested with CLART ${ }^{\circledR}$ CMA MPCR had very low CTC counts to definitively conclude that MPCR is inaccurate for performing genetic analyses on CTC, and a larger sample, preferably of CRC patients with high CTC counts is needed to derive conclusions (Table 4). 
Through previous experiences - data not shown-, and concordant with other authors' reports, our laboratory established the KRAS mutation detection cutoff value (dCt KRAS) using castPCR ${ }^{\mathrm{TM}}$ at 21 , such that mutations with a $\mathrm{dCt}<21$ were considered undoubtedly true mutations $[\mathbf{1 4 , 3 0}]$. According to this, castPCR ${ }^{\mathrm{TM}}$ detected mutations in CTC in 4 out of 8 patients from group 1 -a fifh patient was found to be mutated in CTC but with a dCt=33-, finding that KRAS had changed its status in 3 of them. In the 3 patients tested from group 2 (all KRAS ${ }^{\mathrm{WT}}$ in the primary tumor), castPCR ${ }^{\mathrm{TM}}$ detected KRAS mutations in CTC in 2 of them. Our results using castPCR ${ }^{\mathrm{TM}}$ agree with those reported by the IsoFlux ${ }^{\mathrm{TM}}$ manufacturer using this technology and are actually more robust, as they were performed in a larger number of only-stage IV CRC patients, while the manufacturer has only reported the results of castPCR ${ }^{\mathrm{TM}}$ on CTC in four patients with CRC in stages II and IV [14]. Not suprisingly, in all the patients where the mutation $\mathrm{dCt}$ in CTC was < 21, either the number of CTC (296 CTC in one patient and 203 CTC in another) or the total amount of DNA (98 $\mathrm{ng} / \mu \mathrm{l}$ in the patient with two KRAS mutations in CTC) were especially high (see Table 5), suggesting, as otherwise expected, a better performance of castPCR ${ }^{\mathrm{TM}}$ in samples rich in tumoral DNA.

However, no coincidence was found between the type of mutation in the primary tumor and that in CTC. Of note, in one chemo-naïve KRAS ${ }^{W T}$ patient from group 1, with a very high tumor burden and where IsoFlux ${ }^{\mathrm{TM}}$ counted 203 CTC, castPCR $^{\mathrm{TM}}$ detected the mutation p.G12A [dCt 17.3], probably reflecting an underepresented subpopulation from the primary tumor not detected by TheraScreen ${ }^{\circledR}$. In other patient from group 1 -were only 4 CTC were detected- castPCR ${ }^{\mathrm{TM}}$ found the p.G13D mutation, although with a $\mathrm{dCt}=33$. In this patient primary tumor, TheraScreen ${ }^{\circledR}$ found the p.G12V mutation. A slight cross-reactivity has been described between the probes 
for p.G12S, p.G12D and p.G13D assays but not for the p.G12V mutation, thus making cross-reactivity an improbable explanation (Table 5) [30].

Two of the patients from group 2 found to be KRAS ${ }^{M U T}$ in CTC -both of them KRAS ${ }^{\mathrm{WT}}$ by TheraScreen ${ }^{\circledR}$ in the primary tumor- had been previously treated with chemotherapy plus targeted agents. One of them had indeed received 20 cycles of FOLFIRI-Cetuximab immediately prior to CTC collection at the time of progression. The mutation found in CTC was p.G12A [dCt=19.4], possibly suggesting a change in KRAS status due to treatment selection pressure (Table 5). Indeed, Misale et al [31], showed that resistance to cetuximab could either emerge from the selection of pre-existing KRAS mutant clones or as a result of new, treatment-induced mutations. Likewise, Díaz et al [32] found that metastases of CRC patients treated with the anti-EGFR panitumumab usually harbored resistant clones that expanded rapidly following treatment initiation. The other patient from group 2 found to be KRAS ${ }^{M U T}$ in CTC, had received 5 cycles of FOLFOX-Bevacizumab as first-line treatment, just before the collection of CTC at the time of progression. Interestingly, in this patient, castPCR ${ }^{\mathrm{TM}}$ unveiled two different KRAS mutations, both with a dCt below 21 (p.G12A [10.7], p.G13D [dCt 12]) (Table 5). This patient showed a very high tumor burden, with peritoneal carcinomatosis and hepatic, pulmonary and lymph-node metastases, possibly reflecting a biologically aggressive and heteregenous tumour with different cell clones, not all of them detectable by the standard KRAS mutation tests. Prior treatment with cytotoxic chemotherapy, even though it did not include an anti-EGFR agent, may also have contributed to the mutated KRAS status in CTC, in agreement with previous studies showing that systemic therapy can enhance tumor diversity and confer a more agressive tumor phenotype $[33,34]$. Likewise, prior treatment with bevacizumab in the 
first-line setting could have selected already existing KRAS mutant clones detectable though CTC at the time of progression. Indeed, selection of KRAS mutant clones after exposure to bevacizumab should be the object of in-depth investigations since it may have clinical implications regarding the most appropriate treatment sequence in patients with wild-type RAS advCRC $[35,36]$.

These cases illustrate the advantage of "liquid" over traditional biopsies for acknowledging relevant celullar clones with potential for hematogenous dissemination and eventual metastasis. The mutational information obtained from CTC when castPCR $^{\mathrm{TM}}$ is coupled to an efficient isolation system, allows to better interpret the dynamic heterogeneity of cancer and may eventually serve to take therapeutic decisions. Indeed, the intratumor heterogeneity (ITH) explains the tumor sampling bias that can occur when performing solid biopsies, as they will not inform of cell subpopulations outside the area of biopsy and, when using low-sensitivity methods for mutational analysis, small but biologically important cell subclones may go unrecognized [1].

Our study is limited by the small sample size and the fact that CTC were collected at a single time point. Therefore we could not monitor CTC enumeration and molecular dynamics in a serial manner. It would be of interest to couple the IsoFlux ${ }^{\mathrm{TM}}$ system to other highly sensitive technologies like NGS, ddPCR and BEAMing (Bead Emulsion Amplification and Magnetics) -the latter claimed to have a $0.01-0.001 \%$ sensitivity- for conducting mutational studies on CTC in CRC. To our knowdledge, BEAMing has only been used in the detection of mutated ctDNA and never on CTC, and neither NGS nor ddPCR have ever been used coupled to IsoFlux ${ }^{T M}$ in CRC [24]. Future studies should test the performance of IsoFlux ${ }^{\mathrm{TM}}$ combined with these technologies and, ideally, compare 
the performance of CTC as a predictive tool with that of ctDNA. Finally, these technologies should be used to conduct mutational analyses in solid tumor biopsies in order to unveil their genetic heterogeneity and ideally couple the results to the information provided by liquid biopsies.

\section{Materials and methods}

\section{a. Study design}

This was a prospective-retrospective study, conducted in 3 hospitals in Spain. Group 1 consisted of chemo-naïve patients with advanced CRC, in which blood for CTC was extracted before starting chemotherapy. Patients assigned to group 2 had KRAS ${ }^{\mathrm{WT}}$ advanced CRC progressing to chemotherapy for advanced disease. Blood for CTC was extracted at the time of radiologic progression prior to starting a new line of treatment. CTC enumeration was performed with both CellSearch ${ }^{\circledR}$ and IsoFlux ${ }^{\mathrm{TM}}$ in patients from group 1 and only with CellSearch $\AA$ in patients from group 2. CTC recovery for KRAS mutational analysis was performed in both groups using IsoFlux ${ }^{\mathrm{TM}}$. The study was approved by the review boards of all participating institutions. All patients signed an informed consent before being enrolled in the study.

\section{b. Patients}

Patients were included between November 2013 and October 2015. They had to be $\geq 18$ years of age with histologically confirmed stage IV colorectal adenocarcinoma. Exclusion criteria included having suffered 
another malignancy in the previous 5 years or not fulfilling the inclusion criteria to be assigned to any of the two groups.

\section{c. Study objectives}

The primary objetive was to compare the efficiency in the enumeration of CTC of CellSearch ${ }^{\circledR}$ and IsoFlux ${ }^{\mathrm{TM}}$ in patients from group 1 and to stablish the best CTC cut-off point for predicting survival with each detection system. Secondary objectives were: a) to find differences in CTC counts based on clinical features in each group, b) to study clinical characteristics based on the selected CTC cutoffs in group 1, c) to describe the proportion of patients tested with IsoFlux ${ }^{\mathrm{TM}}$ who achieved $\geq 4 \mathrm{CTC} / 7.5 \mathrm{ml}$ of blood (the established limit of detection (LOD) for genetic studies using castPCR ${ }^{\mathrm{TM}}$ ) in patients from group 1 and d) to test three different PCR-based methods for KRAS mutational analysis in CTC isolated with IsoFlux ${ }^{\mathrm{TM}}$ (cobas ${ }^{\circledR}, \mathrm{CLART}^{\circledR}$, and castPCR ${ }^{\mathrm{TM}}$ ) in both groups.

\section{d. Study procedures}

Peripheral blood samples were collected in triplicate from each patient, from group 1 before starting chemotherapy, and in duplicate before starting a new chemotherapy line in patients from group 2 . The sample processing was centralized at the CTC Laboratory of the Hospital Clínico San Carlos.

\section{i. CTC isolation and enumeration}

For CTC isolation and enumeration with the CellSearch ${ }^{\circledR}$ system (Janssen Diagnostics, LLC) peripheral blood samples 
were collected in $10 \mathrm{~mL}$ CellSave tubes (Janssen Diagnostics, LLC). For isolation and enumeration of CTC with IsoFlux ${ }^{\mathrm{TM}}$ (Fluxion Biosciences Inc, South San Francisco, CA) peripheral blood samples were collected in $10 \mathrm{ml}$ EDTA tubes (BD Vacutainer). In both systems, an immunomagnetic isolation of CTC was done by means of magnetic beads coated with antiEpCAM antibodies. For identification and enumeration of CTC with CellSearch ${ }^{\circledR}$ the Celltracks Autroprep system (Jansenn Diagnostics, LLC) was used, which automatically enriches the sample of CTC, labeling them with fluorescent antibodies directed to cytokeratins (CK8, 18 and 19), 4', 6-diamino-2fenilindol (DAPI) an anti-CD45 antibody. After sample incubation, the Celltracks Analyzer II System (Janssen Diagnostics, LLC), automatically scans the entire surface of the cartridge, acquires images and displays them in a gallery format for final classification. Two different experts classify, then, an event as a tumor cell when its morphological features are consistent with that of a tumor cell and it exhibits the phenotype EpCAM+, CK+, DAPI+ and CD45-. For IsoFlux ${ }^{\mathrm{TM}}$ enumeration, a 10-mL peripheral blood sample was used for the CTC enrichment. A mixture of the cellular portion of blood plus inmunomagnetic beads coated with anti Ep-CAM is loaded onto a microfluidic cartridge and processed with the IsoFlux instrument. Obtained СTC are then fixed and stained with 
fluorescent reagents. These include anti-CK-fluorescein isothiocyanate (FITC) specific for cytokeratine, anti CD45 specific for leucocytes, and Hoechst 33342, which stains the cell nucleus. The sample was examined under a fluorescence microscope by two experts. Cells were scored as CTC if they were $\mathrm{CK}+, \mathrm{CD} 45-$, nucleated, and morphologically intact. Imaging was performed using a fluorescence microscope kindly provided by Izasa Scientific (WerfenLife, L'Hospitalet de Llobregat, Barcelona, Spain).

\section{ii. KRAS mutational analysis on primary tumor and on CTC}

Formalin-fixed paraffin-embedded (FFPE) tumor samples were used for the study of KRAS in the primary tumor. A $10 \mathrm{~mL}$ peripheral blood sample collected in an EDTA tube (BD Vacutainer) was used for the CTC recovery with IsoFlux ${ }^{\mathrm{TM}}$. Whole-genome amplification was performed directly on recovered CTC after cell lysis (REPLI-g UltraFast Mini Kit; Qiagen, Germany). Amplified g-DNA was purified and eluted in $50 \mu \mathrm{L}$ of AE buffer (QIAmp DNA Micro Kit; Qiagen, Germany) in order to perform KRAS mutational analysis with three different PCR-based methods.

\section{a) cobas $®$ and TheraScreen $\circledast$ real-time PCRs}

cobas ${ }^{\circledR}$ KRAS Mutation test for use with cobas ${ }^{\circledR} 4800$ System (Roche Molecular systems Inc.), is a FDA-approved for tissue samples real-time PCR that detects seven somatic 
mutations in codons 12 and 13 of the KRAS gene (G12A, G12D, G12R, G12C, G12S, G12V, G13D). We used it for KRAS mutational analysis in DNA from CTC and from FFPE tumor samples, as previously described [29]. TheraScreen ${ }^{\circledR}$ K-RAS Mutation Kit (QIAGEN Manchester Ltd.) combines ARMS ${ }^{\circledR}$ (amplification refractory mutation system) and Scorpions ${ }^{\circledR}$ technologies for allele specific amplification. It detects the same 7 KRAS mutations mentioned above and is approved by the FDA, also for tissue samples, as a companion diagnostic test for use of cetuximab in KRASWT CRC. Compared to $\operatorname{cobas} \AA$, TheraScreen $\AA$ assay informs not only of KRAS mutational status (mutant versus wild-type), but also of the specific mutation present. We used TheraScreen ${ }^{\circledR}$ assay for KRAS mutational analysis FFPE tumor samples, as previously described [30].

\section{b) CLART ${ }^{\circledR}$ CMA multiplex PCR}

CLARTR CMA multiplex PCR (mPCR) was used for the detection and identification of point mutations in codons 12 and 13 of KRAS (G12A, G12D, G12R, G12C, G12S, G12V, G13D, Q61H(A>T), Q61L), using the CLART ${ }^{\circledR}$ CMA KRASBRAF-PI3K kit (GENOMICA S.A.U, Madrid, Spain). The low density microarray-based CLART ${ }^{\circledR}$ technology and the clinical array reader autoclart ${ }^{\circledR}$ (GENOMICA S.A.U, Madrid, Spain) were used for the visualization of mutations [31]. 


\section{c) Competitive Allele-Specific Taqman PCR (castPCR ${ }^{\mathrm{TM}}$ )}

An analysis of DNA purified from CTC was performed by means of the TaqMan Mutation Detection Assays (ThermoFisher Scientific Inc. Waltham, MA USA). These assays allow to detect somatic mutations while being boosted by the Competitive Allele Specific Technology $\left(\right.$ castPCR ${ }^{\mathrm{TM}}$ ), as previously described [32]. This technology is highly specific and sensitive, being able to detect scarce quantities of mutant DNA -down to $0.5 \%$ of mutant DNA- in a sample containing huge amounts of wild-type DNA. Down to 4 copies of target DNA in a background of 10.000 wildtype cells have been detected elsewere [14]. The Applied Biosystems 7900HT Fast Real-Time PCR System (Life Technologies Corporation, Foster City, CA) was used to detect mutations in codons 12 and 13 of KRAS, as previously described [32].

\section{e. Statistical analysis}

A descriptive analysis was done summarizing quantitative variables by their frequency distribution and qualitative variables by their mean and standard deviation $( \pm S D)$ or their median and range. The $\chi^{2}$ test was used to compare qualitative variables and the Mann-Whitney $U$ test for nonnormally distributed quantitative variables. Spearman's Rho test was used 
for correlation studies and Receiver Operator Characteristics (ROC) analysis was performed to assess the utility of the two CTC detection systems (CellSearch ${ }^{\circledR}$ and Isoflux ${ }^{\mathrm{TM}}$ ) in predicting survival. The area under the curve (AUC) and its $95 \%$ confidence intervals $(95 \% \mathrm{Cl})$ were calculated. Based on the coordinates of the curve, a cut-off point was selected using the Youden's index.

Overall survival (OS), was defined as the time from CTC collection until the date of death or last follow-up. OS was summarized using the Kaplan-Meier method and Cox proportional hazards regression model was used to determine the significance of each enumeration system with their selected cut-offs in the prediction of death. The discrimination of the model was tested by calculating Harrell's C-statistic after fitting the Cox proportional hazards model. The C-statistic (c) is the probability of concordance between observed and predicted survival based on pairs of individuals, with $\mathrm{c}=0.5$ for random predictions and $\mathrm{c}=1$ for a perfectly discriminating model. Statistical significance was established at a $\mathrm{P}$ value $<0.05$. The statistical package Stata for Windows ${ }^{\circledR}$ version 12.0 (StataCorp LP, College Station, TX) was used for all statistical calculations. 


\section{Conclusions}

IsoFlux ${ }^{\mathrm{TM}}$ is more efficient than CellSearch ${ }^{\circledR}$ in the isolation of CTC in patients with advCRC, achieving, in a majority of cases the established minimum of CTC for PCRbased genetic analyses. We propose CTC $\geq 11$ as the reference cutoff point for predicting survival with IsoFlux ${ }^{\mathrm{TM}}$ in advCRC. Finally, we show that castPCR ${ }^{\mathrm{TM}}$ seems a reliable method for performing KRAS mutational studies on DNA from CTC. Together, these results indicate that $\mathrm{CTC}$ isolated with IsoFlux ${ }^{\mathrm{TM}}$ may be a useful prognostic marker as well as a powerful predictive tool in advCRC.

\section{Acknowledgements}

We thank María Luisa Maestro de las Casas for her support in launching this project.

\section{Author Contributions}

Eduardo Díaz-Rubio and Javier Sastre designed the project.

Javier Sastre, Santiago Cabezas-Camarero, Antonio Sánchez-Ruiz, Mariano Provencio and Enrique Aranda recruited the patients for the study.

Virginia de la Orden, Beatriz Mediero-Valeros and Silvia Veganzones-de-Castro, performed CTC isolation for enumeration and KRAS mutational studies.

Santiago Cabezas-Camarero coordinated the project, collected both clinical and laboratory data and analysed the results, the latter in collaboration with Eduardo DíazRubio and Manuel Enrique Fuentes-Ferrer.

Santiago Cabezas-Camarero and Eduardo Díaz-Rubio prepared the manuscript, the final version of which was approved by all the authors.

\section{Conflicts of Interest}

This project was conducted thanks to a grant from Merck KGaA, Darmstadt, Germany 


\section{References}

1. Swanton C. Intratumor heterogeneity: evolution through space and time. Cancer Res 2012;72:4875-82.

2. Ferreira MM, Ramani VC, Jeffrey SS. Circulating tumor cell technologies. Mol Oncol 2016;10:374-94.

3. Masuda $T$, Hayashi N, Iguchi $T$, et al. Clinical and biological significance of circulating tumor cells in cancer. Mol Oncol 2016;10:408-17.

4. Cristofanilli M, Budd GT, Ellis MJ, et al. Circulating tumor cells, disease progression, and survival in metastatic breast cancer. N Engl J Med 2004;351:781-91.

5. Smerage JB, Barlow WE, Hortobagyi GN, et al. Circulating tumor cells and response to chemotherapy in metastatic breast cancer: SWOG S0500. J Clin Oncol 2014;32:3483-9.

6. de Bono JS, Scher HI, Montgomery RB, et al. Circulating tumor cells predict survival benefit from treatment in metastatic castration-resistant prostate cancer. Clin Cancer Res 2008;14:6302-09.

7. Cohen SJ, Punt $\mathrm{CJ}$, lannotti N, et al. Prognostic significance of circulating tumor cells in patients with metastatic colorectal cancer. Ann Oncol 2009;20:1223-29.

8. Sastre J, Maestro ML, Gómez-España A, et al. Circulating tumor cell count is a prognostic factor in metastatic colorectal cancer patients receiving first-line chemotherapy plus bevacizumab: a Spanish Cooperative Group for the Treatment of Digestive Tumors study. Oncologist 2012;17:947-55.

9. Sastre J, Maestro ML, Puente J, et al. Circulating tumor cells in colorectal cancer: correlation with clinical and pathological variables. Ann Oncol 2008;19:935-8.

10. Allen-Mersh TG, McCullough TK, Patel H, et al. Role of circulating tumor cells in predicting recurrence after excision of primary colorectal carcinoma. $\mathrm{Br} J \mathrm{Surg}$ 2007;94:96-105.

11. Sotelo MJ, Sastre J, Maestro ML, et al. Role of circulating tumor cells as prognostic marker in resected stage III colorectal cancer. Ann Oncol 2015;26:535-41.

12. Miller MC, Doyle GV, Terstappen LWMM, et al. Significance of circulating tumor cells detected by the CellSearch system in patients with metastatic breast, colorectal and prostate cancer. J Oncol 2010;2010:617421.

13. Krebs MG, Sloane R, Priest $L$, et al. Evaluation and prognostic signficance of circulating tumor cells in patients with non-small-cell lung cancer. J Clin Oncol 2011;29:1556-63. 
14. Harb W, Fan A, Tran T, et al. Mutational analysis of circulating tumor cells using a novel microfluidic collection device and qPCR assay. Transl Oncol 2013;6:528-38.

15. Alva A, Friedlander T, Clark M, et al. Circulating tumor cells as potential biomarkers in bladder cancer. J Urol 2015;194:790-8.

16. Amato $R$, Brobey $R$, Dehghani $M$, et al. Somatic mutation detection via sequencing using circulating tumor cell samples from patients with renal cell and prostate cancer. http://support.fluxionbio.com/hc/en-us/categories/200172088

17. Ma Y, Luk A, Young FP, et al. Droplet digital PCR based androgen receptor variant 7 (AR-V7) detection from prostate cancer patient blood biopsies. Int J Mol Sci 2016;17. pii: E1264.

18. Wynne JF, Modlin LA, Fan A, et al. Analysis of circulating tumor cells in early stage non-small cell lung cancer patients treated with stereotactic ablative radiotherapy. http://support.fluxionbio.com/hc/en-us/categories/200172088

19. Sánchez-Lorencio MI, Ramirez P, Saenz L, et al. Comparison of two types of liquid biopsies in patients with hepatocellular carcinoma awaiting orthotopic liver transplantation. Tranplant Proc 2015;47:2639-42.

20. Alix-Panabières $C$, Pantel K. Clinical applications of circulating tumor cells and circulating tumor DNA as liquid biopsy. Cancer Discov 2016;6:1-13.

21. Torre LA, Bray F, Siegel RL, et al. Global cancer statistics, 2012. CA Cancer J Clin 2015;65:87-108.

22. Sorich MJ, Wiese MD, Rowland A, et al Extended RAS mutations and anti-EGFR monoclonal antibody survival benefit in metastatic colorectal cancer: a metaanalysis of randomized, controlled trials. Ann Oncol 2015;26:13-21.

23. Stintzing S, Modest DP, Rossius L, et al. FOLFIRI plus cetuximab versus FOLFIRI plus bevacizumab for metastatic colorectal cancer (FIRE-3): a post-hoc analysis of tumour dynamics in the final RAS wild-type subgroup of this randomised openlabel phase 3 trial. Lancet Oncol 2016; doi:10.1016/S1470-2045(16)30269-8.

24. Tabernero J, Lenz HJ, Siena $S$, et al. Analysis of circulating DNA and protein biomarkers to predict the clinical activity of regorafenib and assess prognosis in patients with metastatic colorectal cancer: a retrospective, exploratory analysis of the CORRECT trial. Lancet Oncol 2015;16:937-48.

25. Nicolazzo C, Massimi I, Lotti LV, et al. Impact of chronic exposure to bevacizumab on EpCAM-based detection of circulating tumor cells. Chin J Cancer Res 2015;27:491-96. 
26. http://support.fluxionbio.com/hc/en-us/articles/216408518-IsoFlux-EnhancedEnrichment-Kit-Specification (accessed on 10/09/2016)

27. http://www.accessdata.fda.gov/cdrh_docs/pdf14/P140023c.pdf (accessed on 10/09/2016)

28. https://www.qiagen.com/us/resources/technologies/oncology-companiondiagnostics/therascreen-kras-test-usa-labs/ (accessed on 10/09/2016)

29. http://genomica.es/en/documents/GENOMICA_2014_English.pdf (accessed on 10/09/2016)

30. Didelot A, Le Corre D, Luscan A, et al. Competitive allele specific TaqMan PCR for KRAS, BRAF and EGFR mutation detection in clinical formalin fixed paraffin embedded samples. Exp Mol Pathol 2012;92;275-80.

31. Misale S, Yaeger R, Hobor $S$, et al. Emergence of KRAS mutations and acquired resistance to anti-EGFR therapy in colorectal cancer. Nature 2012;486:532-6.

32. Díaz LA Jr, Williams RT, Wu J, et al. The molecular evolution of acquired resistance to targeted EGFR blockade in colorectal cancers. Nature 2012;486:537-40.

33. Comprehensive genomic characterization defines human glioblastoma genes and core pathways. Nature 2008;455:1061-8.

34. Ding L, Ley $T J$, Larson $D E$, et al. Clonal evolution in relapsed acute myeloid leukaemia revealed by whole-genome sequencing. Nature 2012;481:506-10.

35. Heinemann V, von Weikersthal LF, Decker T, et al. FOLFIRI plus cetuximab versus FOLFIRI plus bevacizumab as first-line treatment for patients with metastatic colorectal cancer (FIRE-3): a randomised, open-label, phase 3 trial. Lancet Oncol 2014;15:1065-75.

36. Schwartzberg LS, Rivera F, Karthaus M, et al. PEAK: a randomised, multicenter phase II study of panitumumab plus modified fluorouracil, leucovorin, and oxaliplatin (mFOLFOX6) or bevacizumab plus mFOLFOX6 in patients with previously untreated, unresectable, wild-type KRAS exon 2 metastatic colorectal cancer. J Clin Oncol 2014;32:2240-7.

(C) 2016 by the authors; licensee Preprints, Basel, Switzerland. This article is an open access article distributed under the terms and conditions of the Creative Commons by Attribution (CC-BY) license (http://creativecommons.org/licenses/by/4.0/). 